\section{Pursuing Value-Based Surgery}

\section{Grant T Fankhauser*}

Department of Vascular Surgery, University of Texas Medical Branch, Galveston, USA

Surgery is a costly part of healthcare, responsible for approximately $30 \%$ of healthcare expenditures [1]. Given the cost of surgical services is it a worthy endeavor to try to maximize the quality of surgical care per dollar spent. In healthcare we tend to define this as value: quality (outcomes) per cost [2]. When it comes to surgery the definition of value is elusive. Inaccurate or haphazard determinants of surgical value may lead to improper decision-making. An accurate and useful measurement of surgical value may give a healthcare organization a distinct advantage [3-5]. Before discussing the development of such a measurement in surgery it is worth mentioning the current legislative and regulatory requirements for quality for US physicians.

In April 2015, the Medicare Access and CHIP Reauthorization Act (MACRA) was signed into law [6]. As part of this legislation, the Sustainable Growth Rate formula in Medicare was abolished and replaced with the Quality Payment Program (QPP) [7]. The Quality Payment Program incorporates and replaces several other previous quality systems such as the Physician Quality Reporting System (PQRS), the Electronic Health Record (EHR) Meaningful Use program, and the Value-Based Modifier program. Medicare payments to physicians under the new system will begin in 2019 but the data collection period began in January of 2017. The Merit-based Incentive Payment System (which the majority of physicians will partake in) includes four components: Quality, Advancing Care Information (ACI), Resource Use (Cost), and Improvement Activities. Each of these components has a number of measures which physicians must meet in order to either receive bonus payments or avoid penalties for non-compliance.

With all of these new regulatory requirements meant to improve healthcare quality, is it safe to assume that surgeons complying with the requirements are delivering value-based surgery? No. Can we at least assume that surgeons complying with MIPS are delivering surgical care of higher value than those not complying? No. Then what should we be measuring to determine if surgeons are performing value-based surgery? That is the crux of the matter.

Before undertaking any quality-improvement project, healthcare organizations must first recognize that the quality and value they need to measure are distinct entities and require a separate process than

*Corresponding author: Grant T Fankhauser, Department of Vascular Surgery, University of Texas Medical Branch, Galveston, USA, Tel: +1 4097726366; E-mail: gtfankha@utmb.edu

Citation: Fankhauser GT (2017) Pursuing Value-Based Surgery. J Surg Curr Trend Innov 1: 001

Received: July 25, 2017; Accepted: August 04, 2017; Published: August 18, 2017

Copyright: (c) 2017 Fankhauser GT. This is an open-access article distributed under the terms of the Creative Commons Attribution License, which permits unrestricted use, distribution, and reproduction in any medium, provided the original author and source are credited. those related to laws or regulations. It would be wonderful if participation in the government's QPP automatically led to value-based delivery of care. But it does not. And it would be wonderful if delivering value-based care led to automatic compliance with the QPP. But it does not. These are two distinct goals with separate processes that happen to share a common theme. The healthcare organizations that recognize this fact and react accordingly are likely to reap the benefits in both processes.

So, why focus on just surgery and not healthcare delivery at-large? First, as a surgeon, it is an area of medicine I can address with authority and one I know well. Second, surgery is a costly part of the healthcare system and one I have personally witnessed is rife with inefficiency. Third, because of the complexities of hospital-surgeon relationships and the multiple phases of care across which surgery operates, the determination (i.e., measurement) of value is especially difficult. Therefore, it is an area where developing an accurate and useful measurement of value could result in large improvements.

The difficulty in determining surgical value resides both in deciding what to measure and whom to measure, and it matters who is measuring. I will illustrate with an example. Take the practice of a single surgeon at a single hospital. For simplicity let us assume that surgeon only performs inguinal hernia repairs. In measuring the value of her surgeries, multiple metrics could be used in numerator of the value equation: success at hernia repair, postoperative length of stay, freedom from perioperative complications, degree of postoperative pain, or lack of hernia recurrence. Just as many metrics could be used in the denominator of the value equation: total operative time, cost of disposable supplies or, cost of postoperative care, number of postoperative visits, cost of complications, or total cost from preoperative visit through surgery to postoperative visit. Any one of those numerators divided by any of the denominators would yield a measurement of value in this surgeon's hernia repair practice. But which is the best equation? And what if the various metrics are not independent but co-dependent or interdependent?

Let us assume that another hernia surgeon at the same hospital performs the same procedure but likes to use a more expensive hernia mesh. The more expensive mesh is easier to use and results in a shorter operative time. The surgeon using the more expensive mesh would have greater value in an equation measuring operative time but less value in an equation measuring supply cost. Let us assume the time saved in the operating room outweighs the cost of the more expensive mesh, but the more expensive mesh leads to a higher rate of recurrent hernia. Let us also assume that the more expensive mesh causes a slightly lower level of postoperative pain and may lead to decreased length of stay. But, the same mesh is associated with a slightly higher risk of a wound infection. The comparison in value between these two surgeons performing the same procedure just became incredibly difficult. And so far the only difference between them is which hernia mesh they use. 
Which surgeon is delivering a higher of value of surgery? This rather simple example demonstrates that the answer is not simple at all. We can see that very quickly the simple value equation becomes one which incorporates many numerators and many denominators, and changes in one can affect the other. We also cannot forget that the value determined is different based on who is measuring.

For the hospital, the most value may be derived from the surgeon with the best outcomes per profit rather than outcomes per cost. A profit equation would factor in such variables as the payer mix of the patients as well as the costs of the operation and postoperative stay along with any complications. But, that equation would not factor in additional revenue (or profit) directed by that physician to the hospital in the form of referrals or tests. And, the profit equation might only consider profit per patient not profit per month or year. If physician two has a higher profit-to-outcome ratio but only performs 10 hernia repairs per year, the hospital may derive greater net value from surgeon one's lower profit-to-outcome ratio through higher volume. For the patient, the quality and outcome portion of the value equation will have a higher relative value. Postoperative pain and hernia recurrence will be more important than cost of the hernia mesh. The value of each surgeon's hernia repair will be calculated differently by the hospital and by the patient. The value would also be different if calculated by the insurance company or by the chair of surgery or by the hospital pharmacy or by the patients' employers. Each entity is likely to measure value differently based on individual priorities. Some will focus on short-term financial issues while others use a longer perspective. Outcome measures such as pain, the ability return to work, and freedom from hernia recurrence are harder to measure (quantify) in order to plug into an equation.
The purpose here is not to answer all of these questions but rather to illustrate that determining value in surgery is incredibly complex. An overall value equation may be impossible to craft but targeted determinations of value are possible when the right questions are asked. We must think carefully which aspects of value we really want to measure, track, and ultimately improve. Improvements in one aspect of value may have negative consequences on another aspect and unforeseen effects on yet another. As we move to maximize value-based surgery we must ensure we know which value we are pursuing.

\section{References}

1. Moore FD (1985) Surgical streams in the flow of health care financing. The role of surgery in national expenditures: what costs are controllable? Ann Surg 201: 132-141.

2. Porter ME (2010) What is value in health care? N Engl J Med 363: 2477 2481.

3. Lee TH (2010) Putting the value framework to work. N Engl J Med 363: 24812483.

4. Yount KW, Turrentine FE, Lau CL, Jones RS (2015) Putting the value framework to work in surgery. J Am Coll Surg 220: 596-604.

5. Porter ME (2009) A strategy for health care reform--toward a value-based system. N Engl J Med 361: 109-112.

6. Congress Gov (2015) Medicare Access and CHIP Reauthorization Act of 2015. 114th Congress (2015-2016), USA.

7. https://qpp.cms.gov 